\title{
LA CALIDAD DEL AGUA DE LOS ACUEDUCTOS DE LAS ÁREAS URBANAS DEL DEPARTAMENTO DEL META, COLOMBIA
}

Flaminio Rueda Camberos ${ }^{1}$, Walter Arboleda Girón ${ }^{2}$, Norton Pérez Gutiérrez ${ }^{3}$

\section{Resumen}

El agua de buena calidad es esencial para la vida y la salud humana. El objetivo de este estudio fue caracterizar los resultados del análisis de calidad de agua de los acueductos de los municipios del departamento del Meta. Se diseñó un estudio descriptivo de los registros sobre la evaluación de calidad del agua para consumo humano, provenientes de acueductos del Meta entre el 2008 y el 2014. Se realizaron 3326 análisis, físico-químicos y microbiológicos, que evidenciaron que solo el 15\% de los registros se encontraban dentro del nivel libre de riesgo, es decir, que se consideran potables o aptos para el consumo humano. El $85 \%$ de los registros restantes se ubicaron en niveles de riesgo que oscilan entre bajo y sanitariamente inviable, lo que significa que el agua no es apta para consumo humano, porque genera riesgo para la salud. Los análisis de calidad del agua de los acueductos adscritos a municipios del departamento del Meta demuestran un bajo cumplimiento de los estándares de calidad para alcanzar las condiciones óptimas para el consumo humano.

Palabras clave: agua, humanos, abastecimiento de agua, agua potable, calidad del agua.

\footnotetext{
${ }^{1}$ Universidad Cooperativa de Colombia, sede Villavicencio. Grupo de Investigación de Villavicencio (GRIVI).

${ }^{2}$ Universidad Cooperativa de Colombia, sede Villavicencio. Grupo de Investigación de Villavicencio (GRIVI).

${ }^{3}$ Universidad Cooperativa de Colombia, sede Villavicencio. Grupo de Investigación de Villavicencio (GRIVI).
} 


\title{
WATER QUALITY OF WATER SUPPLY INFRASTRUCTURE IN URBAN AREAS OF THE DEPARTMENT OF META, COLOMBIA
}

Flaminio Rueda Camberos¹, Walter Arboleda Girón², Norton Pérez Gutiérrez ${ }^{3}$

\begin{abstract}
A good water quality is essential for human life and health. The aim of this research was to characterize the findings of the water quality analysis of the water supply infrastructure in the municipalities of the department of Meta. A descriptive study of the records of drinking water quality of the water supply infrastructure in Meta between 2008 and 2014 was developed. There were carried out 3,326 physicalchemical and microbiological analyzes that showed that only $15 \%$ of the records have non-risk level, therefore, it is considered drinking water for human consumption; the remaining $85 \%$ of records were found in risk level, showing a low water quality, which is not suitable for human consumption, since, it generates risk for health. The analysis of water quality of water supply infrastructure of municipalities of the department of Meta show that there is a low compliance with the water quality standards required to meet the optimal conditions for human consumption.
\end{abstract}




\section{A QUALIDADE DA ÁGUA DOS AQUEDUTOS DAS ÁREAS URBANAS DO DEPARTAMENTO DE META, COLÔMBIA}

Flaminio Rueda Camberos ${ }^{1}$, Walter Arboleda Girón², Norton Pérez Gutiérrez ${ }^{3}$

\section{Resumo}

Água de boa qualidade é essencial para a vida e a saúde humana. O objetivo deste estudo foi caracterizar os resultados da análise da qualidade da água dos aquedutos dos municípios do departamento de Meta. Foi realizado um estudo descritivo dos registros da avaliação da qualidade da água para consumo humano de aquedutos de Meta entre 2008 e 2014. Foram realizadas 3.326 análises físico-químicas e microbiológicas que mostraram que apenas 15\% dos registros estão no nível sem risco e, portanto, apenas esta porcentagem é considerada adequada para consumo humano ou para beber; os restantes $85 \%$ dos registros foram encontrados em níveis sanitários de risco de baixo a inviável, o que não é adequado para consumo humano, pois gera risco à saúde. A análise da qualidade da água dos aquedutos dos municípios do departamento de Meta mostra um baixo cumprimento dos padrões de qualidade para alcançar as condições ótimas para o consumo humano. 


\section{Introducción}

Los seres humanos emplean el agua para múltiples propósitos entre los que se encuentran la agricultura, la industria y el consumo doméstico. Por ello, se hace necesario contar tanto con buenas fuentes de agua, como con la existencia $\mathrm{y}$ buen funcionamiento de sistemas de acueducto que garanticen la continuidad en el suministro el servicio, y la calidad del agua, que es finalmente la garantía de bienestar y de buena salud para la población, en tanto es un vehículo de transmisión de patógenos y microorganismos que puede impactar negativamente la salud de las personas. Según expertos de la Organización Mundial de la Salud (OMS) (1), cerca del 80\% de las enfermedades de los seres humanos están relacionadas con la calidad del agua de consumo.

De acuerdo con el reporte de la OMS (2), se calcula que el $90 \%$ de los 1,8 millones de muertes que ocurren cada año en el mundo por patologías que afectan las vías digestivas, corresponde a niños menores de cinco años. Del mismo modo, la OMS reconoce que el $88 \%$ de las enfermedades diarreicas están relacionadas con factores como la deficiencia de saneamiento e higiene, y el abastecimiento de agua insalubre, razón por la cual al realizar una intervención eficiente de estos factores se prevé una reducción de entre $21 \%$ y $32 \%$ de dicha morbilidad (3).

Durante la Conferencia de las Naciones Unidas realizada en Rio de Janeiro 14 en el año de 1992, se concluyó que "aproximadamente un $80 \%$ de todas las enfermedades y más de una tercera parte de las defunciones en los países en desarrollo son a causa el consumo de agua contaminada $y$, en promedio, hasta una décima parte del tiempo productivo de cada persona, se dedica a las enfermedades relacionadas con el agua" (1).

De acuerdo con los indicadores básicos publicados por el Ministerio de la Protección Social y la Organización Panamericana de la Salud OPS, en el 2011 en Colombia se presentaron 314 muertes por enfermedad diarreica aguda (EDA) en menores de cinco años, con una tasa de 7,34 x por cada 100000 (2).

Según el Ministerio de Salud y de la protección Social, en Colombia en el periodo de 1998 a 2011, las tasas de mortalidad por enfermedad diarreica aguda EDA en menores de cinco años, han disminuido de 33,80 a 3,73 muertes por cada 100000 menores de cinco años (4).

Con respecto al departamento del Meta, la cuarta causa de muerte en los menores de 5 años se relaciona con enfermedades infecciosas y parasitarias, donde la tasa de incidencia para el 2011 fue de 15,48 por cada 100000 habitantes. Para el caso específico de EDA, durante el periodo 2010-2014, se evidenció un descenso de 10 a 6 muertes que equivalen a tasas de incidencia de 11,2 y 6,4 , respectivamente, por cada 100000 menores de 5 años (Tabla 1). Según estas cifras se presenta una tendencia gradual al descenso, sin embargo, la situación sigue siendo crítica en tanto las muertes ocurridas son eventos que, en su mayoría, son evitables (5). 
La prevalencia de EDA en el departamento del Meta es un problema grave de salud pública (Tabla 1) ya que representa la segunda causa de morbilidad, después de la infección respiratoria aguda (IRA). Lo anterior, está íntimamente relacionado con la mala calidad del agua suministrada por la mayoría de acueductos del Meta y con la falta de continuidad del servicio de provisión doméstico.

Basados en los resultados de los análisis de la vigilancia de la calidad del agua para consumo humano de los acueductos del departamento del Meta que realiza la Secretaría de Salud Departamental, se efectuó un estudio descriptivo de la evolución de la calidad del agua para consumo humano en los 30 acueductos de las 29 áreas urbanas del departamento, durante el periodo comprendido entre los años 2008 y 2014 donde se revisa la evolución del Índice de Riesgo de la Calidad del Agua para Consumo Humano por Prestador (IRCApp) a través del tiempo.

El Índice de Riesgo la Calidad del Agua para consumo humano (IRCA), resulta de la valoración ponderada de diferentes parámetros fisicoquímicos y microbiológicos analizados y se implementa en Colombia desde la expedición de la resolución 2115 de 2007 (6).

Las alcaldías municipales son quienes tienen la responsabilidad de prestar el servicio de acueducto en Colombia pudiéndolo ofrecer de forma directa o a través de empresas de servicios públicos ESP, públicas, privadas o mixtas, debidamente legalizadas ante la Superintendencia de Servicios Públicos Domiciliarios quien es el organismo designado para cumplir las funciones de inspección, vigilancia y control de los servicios públicos domiciliarios en el país.

El objetivo de este estudio fue caracterizar los resultados del análisis de calidad de agua de los acueductos de los municipios del departamento del Meta.

\section{Materiales y método}

Se realizó un estudio de tipo cuantitativo, descriptivo y retrospectivo. Se evaluaron los datos desde el año 2008 hasta el 2014.

Se emplearon los resultados producto del análisis de vigilancia de la calidad del agua para consumo humano provenientes de los 30 acueductos de las 29 áreas urbanas del departamento del Meta, realizados por la Secretaría de Salud Departamental. No se incluyeron los datos de los acueductos rurales. Se incluyeron los datos de los acueductos asociados a la Empresa de Acueducto y Alcantarillado de Villavicencio (EAAV) y el promedio de los otros acueductos urbanos se contó como un solo acueducto.

El estudio fue realizado en el departamento del Meta, ubicado en la región centro - oriental de Colombia que cuenta con una extensión de 85770 kilómetros cuadrados, y una población de 945038 habitantes, de los cuales, el $75,68 \%$ viven en el área urbana. Por su parte, el 62,93\% del total de la población está concentrada en su capital, Villavicencio.

Para cada muestra de agua analizada se 
de enfermedades relacionadas con el no cumplimiento de las características físicas, químicas y microbiológicas para el agua de consumo humano (7).

Para el análisis de la calidad del agua en las áreas urbanas del departamento del Meta se utilizaron únicamente los valores promedio anuales por prestador (IRCApp), calculados a partir de los IRCA por muestra de los 3326 resultados de los análisis físico-químicos y microbiológicos de muestras de agua para consumo humano, tomadas de los puntos de muestreo instalados sobre las redes de distribución de los acueductos de las áreas urbanas del departamento del Meta.

Los acueductos rurales, comunitarios y privados de las áreas urbanas se excluyeron por no tener la información completa, por lo tanto, no se posee la cantidad suficiente de registros para generar un análisis estadístico sin sesgo; además, la Secretaría de Salud del Meta informa que estos acueductos no tienen plantas o algún sistema de potabilización y que la gran mayoría suministra a sus usuarios agua no apta para consumo humano.

\section{Materiales e instrumentos para la recolección de datos y muestras}

Las muestras de agua para consumo humano fueron tomadas en puntos definidos de cada uno de los acueductos según lo establecido en la normatividad (6). La programación del muestreo fue definida por las secretarias de salud departamental y de Villavicencio, y es ejecutada por sus técnicos de saneamiento.
Estas muestras son analizadas en el laboratorio de la Secretaría de Salud del departamento del Meta dando cumplimiento a las normas, procedimientos y protocolos internacionales de análisis fisicoquímicos y microbiológicos de agua (8).

El número de muestras, la frecuencia y las características a analizar fueron definidos en la resolución 2115 de 2007 de acuerdo con la población servida de cada acueducto (6).

Las características analizadas fueron: $\mathrm{pH}$, turbiedad, color aparente, alcalinidad, conductividad, cloro residual libre, coliformes totales, concentración de aislamiento de Escherichia coli, bacterias aeróbicas, hierro total, sulfatos y cloruros. Los resultados son exportados a una base de datos predeterminada para calcular el IRCA de la muestra, la cual se imprime posteriormente para reporte. Los datos de análisis son exportados de esta base de datos a una hoja electrónica de Excel para la respectiva evaluación estadística.

De los 3326 resultados obtenidos de los análisis físico-químicos $\mathrm{y}$ microbiológicos, se utilizaron los valores del IRCA por muestra y se calcularon los promedios anuales del IRCApp para su análisis posterior, lo que se resume en la Tabla 2.

\section{Resultados}

Los resultados de los IRCApp promedio anual se obtuvieron a partir del cálculo del promedio simple de los IRCA por muestra de cada acueducto, y el resul- 
tado obtenido, junto con el número de muestras, se presenta en la Tabla 2.

Estos valores del IRCA muestran el nivel de riesgo acorde con la norma (6) y se tabulan en la Tabla 3.

En la Figura 1 se aprecia el número de muestras analizadas por año y la tendencia del IRCApp promedio anual para el total de los 30 acueductos, el cual se encontró dentro del rango de $44.70 \%$, en el año 2008, a 23.10\% en el año 2010.

El número de muestras analizadas se incrementó año a año pasando de 185 en el 2008 a un total de 780 en el 2014, lo que significó un aumento considerable; con esto se superó el número mínimo de muestras establecidas para la vigilancia de la calidad del agua para consumo humano suministrada por los acueductos en Colombia, según la norma mencionada anteriormente.

El acueducto de la EAAV se mantuvo en estado "Sin riesgo" durante todo el periodo de estudio así como los de Restrepo y Puerto Rico.

Seguidos por los acueductos de Acacias, Cumaral, Puerto Lleras, San Carlos de Guaroa y los otros acueductos urbanos de Villavicencio, sin incluir la EAAV, se encontraron durante dos o tres años en nivel de riesgo "Sin riesgo", lo que significa que durante cerca del 29 y el $43 \%$ del periodo analizado suministraron agua apta para consumo humano.

Los acueductos de Barranca de Upia, Castilla la Nueva, El Castillo, Fuentedeoro, Guamal, La Macarena, Lejanías,
Mesetas, Puerto Concordia, Puerto Gaitán, San Martín y Uribe, se encontraron en el nivel "Sin riesgo" durante solo un año, razón por la cual únicamente durante el 14\% del periodo analizado suministraron agua apta para consumo humano a sus usuarios.

En contraste, se observó que los acueductos de Cubarral, Calvario, El Dorado, Granada, Mapiripán, Puerto López, San Juan de Arama, San Juanito y Vista Hermosa no lograron durante los siete años de estudio el nivel "Sin riesgo", lo que significa que durante todo el periodo analizado suministraron agua no apta para consumo humano a sus usuarios.

Por último, los acueductos de Cubarral, El Castillo, Granada, Lejanías y Mesetas se encontraron en nivel de riesgo "Inviable sanitariamente" durante un año del periodo analizado, poniendo en grave riesgo la salud de sus usuarios.

En la Tabla 4 se muestra el resumen de niveles de riesgo por medición y el porcentaje con respecto al total de registros de los acueductos de las áreas urbanas en el periodo de 2008 a 2014, lo que se grafica en la Figura 2 en donde se puede apreciar que solo 29 de los registros (15\%) se encontraron en nivel sin riesgo, 44 registros (22\%) en nivel de riesgo bajo, 49 registros (25\%) en nivel de riesgo medio, 70 registros (36\%) en nivel de riesgo alto y 5 registros en nivel de riesgo "Inviable Sanitariamente".

El análisis de la información se hizo para cada uno de los acueductos de tal manera que se pudieran comparar entre sí, 
presentándose en la Tabla 5 y de manera gráfica en la Figura 3.

\section{Discusión}

El agua es fundamental para la vida $y$ los asentamientos humanos. Países como Estados Unidos tienen buenos resultados de la evaluación de la calidad del agua tanto en zonas urbanas como rurales (1). La contaminación de estas fuentes con residuos fecales es un problema de salud pública que ocasiona enfermedades y puede propagar, incluso, la información genética de microorganismos a la resistencia de los antimicrobianos (9). Adicionalmente, estas pueden ser portadoras de metales que no son aptos para la exposición humana en cantidades considerables por lo cual requieren monitorización de sus concentraciones (10). Incluso el agua embotellada puede ser contaminada y servir como fuente de propagación de infecciones (11).

Más aun, la exposición a sustancias involucradas en el cáncer gastrointestinal como los nitratos puede darse a través del agua como se ha demostrado en algunos países como Indonesia (12) y por ello, son motivo de vigilancia en otros países como Filipinas (13).

Usualmente se evalúan los sitios de procesamiento y depósito de agua, sin embargo, es importante considerar que la calidad del agua puede ser impactada también en las líneas de distribución, debido a la liberación de material de las tuberías, la formación y liberación de

18 biopelícula, fugas, filtraciones y contaminación externa, así como acumula- ción y suspensión de depósitos $(14,15)$. Estas condiciones son favorecidas por la discontinuidad de flujo de líquido en las líneas de distribución, tal y como como se ha demostrado en Panamá, situación que suele ocurrir en las regiones del Meta (16).

Una de las metas de la OMS apunta a que el $90 \%$ de la población mundial pueda acceder al agua potable (2). Este informe reveló que entre 1990 y 2010 fallecieron en Colombia el 19\% de los niños menores de 5 años. En contraste con esta cifra, el promedio regional de mortalidad infantil de menores de 5 años fue de 18\%, mientras que la cifra para los Estados Unidos fue del 8\% del 6\% para Canadá. De igual forma, se evidenció que el $8 \%$ de la población de la región no tiene acceso al agua potable, mientras que en Estados Unidos y Canadá este porcentaje es menor al 2\%. Asimismo, el gasto en salud en Colombia durante el 2009 fue de 392 dólares per cápita, mientras que en Estados Unidos fue de 7860 y en Canadá de 4519. Por ello, no es de extrañar que la esperanza de vida al nacer en Colombia, si bien ha pasado de 71 a 77 años entre 1990 a 2009, en Canadá varió de 77 a 81 y en Estados Unidos de 75 a 79. La probabilidad de morir antes de los 5 años en Colombia varió de 37 a 27 por cada 1000 nacidos vivos, mientras que en Canadá se redujo de 8 a 6 , y en Estados Unidos de 11 a 9. Si bien la mortalidad por diarrea en Colombia se redujo del 5 al 4\%, entre 2000 y 2010, en los otros dos países fue de 0 .

Esta organización plantea que se requiere la evaluación de la seguridad del agua en todas las etapas del siste- 
ma de abastecimiento, desde la cuenca de captación hasta su distribución final al consumidor (17); lo anterior incluye, además, visitas a las instalaciones y evaluación de los conocimientos y opinión de los involucrados en el procesamiento.

En la revisión bibliográfica se encontraron artículos que revelan los resultados de estudios que evalúan la calidad del agua, como el del caso de Ecuador (18), con resultados que revelan diferencias de calidad entre la época seca y lluviosa, donde a pesar de ello, el agua continua siendo aceptable para el consumo humano. Países como Australia, también han desarrollado un índice de riesgo y guías para la provisión de agua para el consumo humano a proveedores públicos y privados, incluso para áreas remotas y comunidades aborígenes (19). Del mismo modo, en los acuíferos urbanos del Valle de Puebla, México, se monitoriza la calidad del agua con un índice de calidad del agua (ICA) siguiendo el método del Consejo del Ministerio del Medio Ambiente de Canadá con lo cual han podido monitorizar el deterioro de la calidad de aquellos explotados intensivamente (17).

Colombia es un país rico en fuentes hídricas (20). Aun así, la prevalencia de enfermedades asociadas al consumo de agua es alta, entre ellas, aquellas enfermedades prevenibles como la diarrea (21). La metodología para la evaluación de la calidad del agua y el riesgo de esta fue establecida en la normatividad colombiana (7) y el desarrollo de indicadores ha sido discutido en publicaciones académicas (22). Países como México tienen normas similares (23) así como la Organización Mundial de la Salud $(24,25)$. Adicionalmente, se han publicado artículos que incluyen como parte de los derechos humanos y como obligación del Estado (26) el acceso a la salud, al agua potable y al saneamiento, donde se relatan los ejemplos de Argentina y de Colombia $(27,28)$. Sin embargo, estos sistemas están en peligro ante la dinámica social precaria de varias poblaciones colombianas como es el caso de la región del Chocó (27).

Se encuentran pocos estudios en la literatura colombiana que evalúan la calidad del agua $(21,29,30)$. Algunos autores buscan asociación entre la contaminación del agua con factores de riesgo de cáncer gástrico, lo anterior mediante investigación cualitativa (21). Este tipo de enfoque ha demostrado que a pesar de la percepción de la problemática del agua, aún existen posibilidades de mejoramiento en la gestión del conocimiento en empresas dedicadas al manejo del agua (30).

Igualmente, un estudio en escuelas rurales ha implementado estrategias exitosas de educación en higiene, para la disminución de enfermedades como la diarrea y el dengue (31). Otros estudios han sido realizados para la medición de la calidad de agua lluvia con el propósito de ser usada en acueductos como en el caso de Ibagué $(31,32)$. Una experiencia que evaluó la calidad del agua en un municipio de Cundinamarca encontró valores de alto riesgo debido principalmente a la presencia de coliformes (33). Más aun, un estudio nacional demostró la presencia de virus entéricos en acueductos en todo el territorio nacional 
desde el 2010 hasta el 2014 (34,35). Por otro lado, la evaluación de una fuente de agua en Norte de Santander demostró ser apta para tratamiento de potabilización (36).

Los datos del presente estudio revelan que la calidad del agua suministrada a la población urbana de los municipios del departamento del Meta, está en un IRCapp alto y medio $(44,7 \%$ y $23,1 \%$ en el año 2008 y 2014 respectivamente), lo cual quiere decir que esta no es apta para el consumo humano, a pesar de presentar evidencias mejoría en la calidad.

En la Tabla 6 se muestran los niveles de riesgo de los acueductos de las áreas urbanas en el periodo de 2008 a 2014. Con base en un código de colores, donde se destaca el nivel de riesgo "Sin riesgo", en azul, se evidencia que el agua apta para consumo humano fue suministrada únicamente por el acueducto de la EAAV, empresa que dio cumplimiento a los estándares de calidad durante todo el periodo del estudio.

Por su parte, los acueductos de Restrepo y Puerto Rico se ubicaron durante 5 años en nivel de riesgo "Sin riesgo" donde suministraron agua apta para consumo humano a sus usuarios durante $71 \%$ del periodo analizado. El resto de municipios alcanzaron niveles aptos por debajo de este periodo de tiempo.

Los resultados se ordenaron de menor a mayor por el valor del IRCApp promedio anual para cada acueducto, lo que muestra que solo el valor promedio

20 la EAAV se ubicó en el nivel de riesgo "Sin riesgo" suministrando agua apta para consumo humano a sus usuarios durante todo el periodo analizado.

De acuerdo con el valor del IRCApp promedio anual, los 29 acueductos restantes se distribuyeron, según nivel de riesgo, de la siguiente manera: seis [6] en nivel de riesgo bajo, doce [12] en nivel de riesgo Medio y once [11] en nivel de riesgo Alto, lo que, según la norma Colombiana, significa que suministraron agua no apta para consumo humano.

En el estudio se encontró que solo 29 de los registros, el $15 \%$, están en nivel sin riesgo, es decir, que distribuyen agua apta para el consumo humano. Los restantes 168 registros, el 85\%, demostraron que el agua no es apta para el consumo humano.

El estudio tiene limitaciones por su naturaleza descriptiva y retrospectiva, donde no se evalúa el impacto en los indicadores de salud humana; sin embargo, arroja información de importancia que es funcional para la toma de decisiones.

\section{Conclusiones}

El estudio de la calidad del agua para el consumo humano es una herramienta para la monitorización del riesgo de transmisión de enfermedades. Esta medición debe ser realizada periódicamente y divulgada a fin de tomar acciones tempranas a fin de implementar medidas para mejorar.

En este estudio se encuentra que solo la EAAV demuestra un cumplimiento 
de suministro de agua potable para consumo humano.

Los demás 29 acueductos de las áreas urbanas, según el valor del IRCApp promedio anual, suministraron agua no apta para consumo humano incumpliendo la norma Colombiana que exige que, de manera continua y en todos los puntos de la red de distribución, se garantice el suministro de agua apta para el consumo humano, lo que puede haber traído consecuencias como altas tasas de enfermedad diarreica, parasitismo y desnutrición en la población.

\section{Referencias}

1. Strosnider $H$,. Kennedy C,. Monti M,. et al. Rural and urban differences in air quality, 2008-2012, and community drinking water quality, 2010-2015. United States. MMWR Surveill Summ. 2017 Jun;66(13):1-10.

2. Organización Mundial de la Salud. Estadísticas Sanitarias Mundiales 2012. Ginebra (Suiza): Organización Mundial de la Salud; 2012. 180 p.

3. Instituto Nacional de Salud. Protocolo de Vigilancia en Salud Publica: Mortalidad por enfermedad diarreica aguda en $<5$ años (EDA). Bogotá, D.C.: Instituto Nacional de Salud; 2014.

4. Ministerio de Salud y Protección Social. Informe nacional sobre la calidad del agua para consumo humano. Bogotá, D.C.; 2013.

5. Secretaría de Salud del Meta. Análisis de la situación de salud con el modelo de los determinantes sociales de salud. Villavicencio; 2014.

6. Palacio Betancourt D,. Lozano Ramirez J. Por medio de la cual se señalan características, instrumentos básicos y frecuencias del sistema de control y vigilancia para la calidad del agua para consumo humano. Resolución 2115 Ministerio de la Protección Social, Ministerio de
Ambiente, Vivienda y Desarrollo Territorial; 2007. 23 p.

7. Ministerio de la Proteccion Social. Por el cual se establece el sistema para la protección y control de la calidad del agua para consumo humano. Decreto 1575 Colombia: Ministerio de Salud y Protección Social; 2007. 14 p.

8. American Public Health Association, American Water Works Association, Water Environment Federation. Standard methods for the examination of water and wastewater; 1999.

9. Gomes Freitas D., Silva R., Bataus L, et al. Bacteriological water quality in school's drinking fountains and detection antibiotic resistance genes. Ann Clin Microbiol Antimicrob [Internet]. 2017 feb [citado 2016 agosto 5] ;16(1):5. Disponible en https:// ann-clinmicrob.biomedcentral.com/articles/10.1186/s12941-016-0176-7

10. Sener S., Sener E., Davraz A. Assessment of groundwater quality and health risk in drinking water basin using GIS. J Water Heal. 2017;15(1):112-32.

11. Georgieva V., Dimitrova Y. Study of the microbiological quality of bulgarian bottled water in terms of its contamination with Pseudomonas Aeruginosa. Centr Eur J Public Heal. 2016;24(4):326-30. 
12. Fathmawati., Fachiroh J., Gravitiani E., et al. Nitrate in drinking water and risk of colorectal cancer in Yogyakarta (Indonesia). J Toxicol Env Heal A. 2017;80(2):120-8.

13. Lomboy M., Riego de Dios J., Magtibay $\mathrm{B}$., et al. Updating national standards for drinking-water: a Philippine experience. J Water Heal. 2017;15(2):288-95.

14. Liu G., Zhang Y., Knibbe W-J., et al. Potential impacts of changing supply-water quality on drinking water distribution: $A$ review. Water Res. 2017;116:135-48.

15. Calderon JA., Rios JF., Echeverria F., et al. Diseño de un sistema piloto para el estudio de la corrosión del material de tubería y su contribución en el deterioro de la calidad del agua potable. Rev Fac Ing. 2008;43:102-13.

16. Erickson JJ., Smith CD., Goodridge A., Nelson KL. Water quality effects of intermittent water supply in Arraijan (Panama). Water Res. 2017;114:338-50.

17. Salcedo E., Garrido S., Esteller M., Martínez M. Application of water quality index to evaluate groundwater quality (temporal and spatial variation) of an intensively exploited aquifer Puebla valley, Mexico. Env Monit Assess. 2016;188(10):573.

18. Baque R., Simba L., Gonzalez B., Suatunce P., Diaz-Ocampo E, CadmeArevalo L, et al. Calidad del agua destinada al consumo humano en un cantón de Ecuador. Rev Cienc UNEMI. 2016;9(20):109-17.

19. Byleveld P., Leask S., Jarvis L., Wall K, Henderson W, Tickell J. Safe drinking water in regional NSW, Australia. Public Helath Res Pr. 2016;26(2).

20. Pena Guzman C., Melgarejo J., Prats D. El ciclo urbano del agua en Bogota, Colombia: estado actual y desafios para la sostenibilidad. Tecnol y Ciencias del 22 Agua. 2016;II(6):57-71.
21. Guzman BL., Nava G., Diaz P. La calidad del agua para consumo humano y su asociación con la morbimortalidad en Colombia, 2008-2012. Biomédica. 2015;35(2):177-90.

22. Amezquita C., Perez A., Torres P. Evaluacion del riesgo en sistemas de distribucion de agua potable en el marco de un plan de seguridad del agua. Rev EIA. 2014;11(21):157-69.

23. Olaiz Fernandez G. Norma oficial mexicana NOM-127-SS!-1994, "Salud ambiental, agua para uso y consumo humano - limites permisibles de calidad y tratamientos a que debe someterse el agua para su potabilizacion. Méjico: Secretaria de Salud; 1995.

24. Organizacion Mundial de la Salud. Guias para la calidad del agua potable. Ginebra, Suiza: Organización Mundial de la Salud; 2006. 408 p.

25. Organización Panamericana de la Salud. Control de la calidad del agua potable en sistemas de abastecimiento para pequenas comunidades. En: Guías para la calidad del agua potable. Washington, D.C.: Organización Panamericana de la Salud; 1998. 132 p.

26. Maqueda Fourcade S. El derecho a la salud y el derecho al agua potable y al saneamiento. Estudio de sus relaciones mutuas y de su protección internacional, con especial análisis de sus implicancias para los pueblos indígenas. Rev Int Derechos Hum. 2012;2(2):16786.

27. Samboni N., Carvajal Y, Escobar J. Revisión de parámetros fisicoquimicos como indicadores de calidad y contaminación del agua: Indicadores de calidad y contaminacion del agua. Rev Ing e Investig. 2007;27(3):172-81.

28. Motta R. El derecho al agua potable en la jurisprudencia colombiana. Rev Repub. 2011;11:53-67. 
29. Avila S, Estupinan S, Diaz L. Calidad bacteriológica del agua vereda El Charco, San Miguel de Sema, Boyacá (Colombia). Nova. 2016;13(25):139-45.

30. Guzman B, Nava T, Bevilacqua P. Vigilancia de la calidad del agua para consumo humano en Colombia: desafíos para la salud ambiental. Rev Fac Nac Salud Pública. 2016;34(2):175-83.

31. Overgaard H., Alexander N., Jaramillo J., et al. Control de diarrea y dengue en escuelas primarias rurales de Colombia: Protocolo de estudio para un ensayo aleatorio y controlado por conglomerados. Rev Salud Bosque. 2015;4(1):7590.

32. Ramirez Arcila H, Ospina Zuniga O. Evaluación de la calidad del agua de lluvia para su aprovechamiento y uso doméstico en la ciudad de Ibagué, Tolima, Colombia. Ing Solidar. 2014;10(17):125-38.
33. Arias B, Bejarano D, Zafra C. Mapa de riesgos para la calidad del agua en sistemas de abastecimiento municipales. Un caso colombiano. Rev Inge@UAN. 2014;4(8):55-68.

34. Pelaez D., Guzman BL., Rodriguez J., et al. Presencia de virus entéricos en muestras de agua para el consumo humano en Colombia: desafíos de los sistemas de abastecimiento. Biomedica. 2016;36(S2):169-78.

35. Baez PA., Jaramillo CM., Arismendi L., et al. Evidencia de circulación del virus de la hepatitis $A$, subgenotipo IA, en muestras ambientales en Antioquia, Colombia. Biomedica. 2016;36(S2):135-47.

36. Miranda R., Ramirez R., Sanchez E. Evaluación de la calidad del agua para consumo humano mediante indicadores fisicoquimicos y microbiologicos en el rio Algodonal. Ingenio UFSPO. 2016;9:131-41. 


\section{Anexos}

Tabla 1. Morbimortalidad por EDA en menores de 5 años en el Departamento del Meta en el periodo 2010 a 2014.

\begin{tabular}{|c|c|c|c|c|c|c|c|c|c|c|}
\hline \multirow{2}{*}{$\begin{array}{c}\text { Comparativo } \\
\text { años } \\
2010-2014\end{array}$} & \multicolumn{5}{|c|}{ Casos } & \multicolumn{5}{|c|}{$\begin{array}{l}\text { Tasas de incidencia por } 100000 \\
<5 \text { años }\end{array}$} \\
\hline & 2010 & 2011 & 2012 & 2013 & 2014 & 2010 & 2011 & 2012 & 2013 & 2014 \\
\hline $\begin{array}{l}\text { Mortalidad por } \\
\text { EDA }<5 \text { años }\end{array}$ & 10 & 10 & 5 & 8 & 6 & 11,2 & 11,06 & 5,5 & 8,7 & 6,4 \\
\hline $\begin{array}{l}\text { Morbilidad por } \\
\text { EDA }<5 \text { años }\end{array}$ & 21052 & 22803 & 19724 & 21856 & 20287 & 235,2 & 252,2 & 216,0 & 237,2 & 218,1 \\
\hline
\end{tabular}

Fuente: Oficina de Vigilancia de la Secretaría de Salud del Meta, 2015.

Tabla 2. Número de muestras analizadas por año y valores del IRCApp promedio anual basados en los resultados de los análisis físicos químicos y microbiológicos del agua de los acueductos de las 29 áreas urbanas del departamento del Meta, realizados por la Secretaría de Salud del Departamento.

\begin{tabular}{|c|c|c|c|c|c|c|c|c|c|c|c|c|c|c|}
\hline \multicolumn{15}{|c|}{$\begin{array}{l}\text { Resumen número de muestras por año y del IRCA(\%)por persona prestadora promedio anual } \\
\text { de los acueductos de las cabeceras municipales del Departamento } \\
\text { del Meta del año } 2008 \text { al } 2014\end{array}$} \\
\hline \multirow{2}{*}{$\begin{array}{l}\text { Acueductos } \\
\text { cabeceras } \\
\text { municipales }\end{array}$} & \multicolumn{2}{|c|}{2008} & \multicolumn{2}{|c|}{2009} & \multicolumn{2}{|c|}{2010} & \multicolumn{2}{|c|}{2011} & \multicolumn{2}{|c|}{2012} & \multicolumn{2}{|c|}{2013} & \multicolumn{2}{|c|}{2014} \\
\hline & $\begin{array}{l}\text { No } \\
\text { Ms }\end{array}$ & \begin{tabular}{|c} 
IRCA- \\
pp
\end{tabular} & $\begin{array}{l}\text { No } \\
\text { Ms }\end{array}$ & \begin{tabular}{|c} 
IRCA- \\
pp
\end{tabular} & $\begin{array}{l}\text { No } \\
\text { Ms }\end{array}$ & \begin{tabular}{|c} 
IRCA- \\
pp
\end{tabular} & $\begin{array}{l}\text { No } \\
\text { Ms }\end{array}$ & \begin{tabular}{|c} 
IRCA- \\
pp
\end{tabular} & $\begin{array}{l}\text { No } \\
\text { Ms }\end{array}$ & \begin{tabular}{|c} 
IRCA- \\
pp
\end{tabular} & $\begin{array}{l}\text { No } \\
\text { Ms }\end{array}$ & \begin{tabular}{|c} 
IRCA- \\
pp
\end{tabular} & $\begin{array}{l}\text { No } \\
\text { Ms }\end{array}$ & \begin{tabular}{|c} 
IRCA- \\
pp
\end{tabular} \\
\hline Acacias & 33 & 8,8 & 16 & 9,31 & 42 & 2,4 & 54 & 5,4 & 51 & 6,3 & 75 & 5 & 88 & 11,00 \\
\hline Barranca & 3 & 36,7 & 0 & & 12 & 226 & 9 & 25,13 & 7 & 30,8 & 15 & 15,6 & 15 & 14,09 \\
\hline Cabuyaro & 2 & 76,1 & 0 & & 8 & 67,6 & 14 & 14,2 & 8 & 35,9 & 23 & 18,5 & 16 & 16,17 \\
\hline Castilla & 4 & 14 & 8 & 26,45 & 11 & 54,3 & 12 & 5,7 & 10 & 9 & 16 & 7,7 & 18 & 4,39 \\
\hline Cubarral & 7 & 51,8 & 10 & 64,56 & 13 & 73,9 & 10 & 70,5 & 11 & 74,1 & 16 & 78,5 & 18 & 80,64 \\
\hline Cumaral & 12 & 39,8 & 0 & & 24 & 8,9 & 22 & 7,9 & 10 & 3,4 & 34 & 4 & 31 & 12,02 \\
\hline El Calvario & 3 & 41,5 & 6 & 54,32 & 11 & 39,8 & 10 & 47,2 & 7 & 41,8 & 15 & 42,4 & 16 & 49,52 \\
\hline El Castillo & 3 & 51,6 & 0 & & 8 & 65,1 & 10 & 70,7 & 11 & 76,9 & 15 & 69,9 & 18 & 82,04 \\
\hline El Dorado & 3 & 56,3 & 6 & 47,95 & 10 & 15,5 & 9 & 37,4 & 10 & 37 & 15 & 22,9 & 18 & 6,63 \\
\hline Fuentedeoro & 6 & 70,5 & 7 & 19,50 & 10 & 26,5 & 12 & 11,4 & 11 & 11,2 & 14 & 4,4 & 17 & 9,71 \\
\hline Granada & 6 & 626 & 4 & 60,43 & 11 & 80,7 & 11 & 69,5 & 12 & 33 & 16 & 27,4 & 17 & 19,55 \\
\hline Guamal & 5 & 72 & 9 & 27,94 & 13 & 128 & 13 & 11,7 & 11 & 7,2 & 16 & 27 & 17 & 7,77 \\
\hline La Macarena & 2 & 44,9 & 0 & & 5 & 29,9 & 11 & 23,1 & 10 & 37,6 & 16 & 34,6 & 15 & 23,29 \\
\hline Lejanías & 5 & 39,7 & 3 & 0,00 & 6 & 10,2 & 9 & 29,9 & 9 & 15,5 & 15 & 26,8 & 17 & 81,03 \\
\hline Mapiripan & 2 & 44 & 6 & 66,57 & 10 & 79,8 & 11 & 26,1 & 9 & 10 & 14 & 15,2 & 3 & 13,78 \\
\hline Mesetas & 6 & 65,5 & 0 & & 9 & 61,9 & 11 & 70,7 & 10 & 70,2 & 15 & 84,1 & 17 & 70,58 \\
\hline Puerto Concordia & 2 & 324 & 0 & & 10 & 7,1 & 8 & 5,1 & 8 & 7,8 & 13 & 9,5 & 12 & 12,71 \\
\hline Puerto Gaitán & 9 & 422 & 0 & & 12 & 20,3 & 9 & 16 & 8 & 13,9 & 17 & 24,2 & 17 & 27,49 \\
\hline Puerto Lleras & 3 & 63 & 0 & & 11 & 222 & 10 & 0,9 & 8 & 3,2 & 14 & 6,3 & 18 & 11,12 \\
\hline Puerto López & 5 & 44,6 & 11 & 64,18 & 29 & 43,3 & 22 & 43,9 & 20 & 36,8 & 32 & 48,5 & 38 & 47,92 \\
\hline Puerto Rico & 2 & 37,6 & 4 & 5,08 & 11 & 4,1 & 9 & 2,2 & 8 & 0 & 10 & 10,8 & 18 & 4,81 \\
\hline
\end{tabular}


Continuación Tabla 2. Número de muestras analizadas por año y valores del IRCApp promedio anual basados en los resultados de los análisis físicos químicos y microbiológicos del agua de los acueductos de las 29 áreas urbanas del departamento del Meta, realizados por la Secretaría de Salud del Departamento.

\begin{tabular}{|c|c|c|c|c|c|c|c|c|c|c|c|c|c|c|}
\hline \multicolumn{15}{|c|}{$\begin{array}{l}\text { Resumen número de muestras por año y del IRCA(\%)por persona prestadora promedio anual } \\
\text { de los acueductos de las cabeceras municipales del Departamento } \\
\text { del Meta del año } 2008 \text { al } 2014\end{array}$} \\
\hline \multirow{2}{*}{$\begin{array}{l}\text { Acueductos } \\
\text { cabeceras } \\
\text { municipales }\end{array}$} & \multicolumn{2}{|c|}{2008} & \multicolumn{2}{|c|}{2009} & \multicolumn{2}{|c|}{2010} & \multicolumn{2}{|c|}{2011} & \multicolumn{2}{|c|}{2012} & \multicolumn{2}{|c|}{2013} & \multicolumn{2}{|c|}{2014} \\
\hline & $\begin{array}{l}\text { No } \\
\text { Ms }\end{array}$ & $\begin{array}{l}\text { IRCA- } \\
\text { pp }\end{array}$ & $\begin{array}{l}\text { No } \\
\text { Ms }\end{array}$ & $\begin{array}{l}\text { IRCA- } \\
\text { pp }\end{array}$ & $\begin{array}{l}\text { No } \\
\text { Ms }\end{array}$ & $\begin{array}{l}\text { IRCA- } \\
\text { pp }\end{array}$ & $\begin{array}{l}\text { No } \\
\text { Ms }\end{array}$ & $\begin{array}{l}\text { IRCA- } \\
\text { pp }\end{array}$ & $\begin{array}{l}\text { No } \\
\text { Ms }\end{array}$ & $\begin{array}{l}\text { IRCA- } \\
\text { pp }\end{array}$ & $\begin{array}{l}\text { No } \\
\text { Ms }\end{array}$ & $\begin{array}{l}\text { IRCA- } \\
\text { pp }\end{array}$ & $\begin{array}{l}\text { No } \\
\text { Ms }\end{array}$ & $\begin{array}{l}\text { IRCA- } \\
\text { pp }\end{array}$ \\
\hline Restrepo & 8 & 18 & 7 & 0,73 & 11 & 0,1 & 11 & 10,1 & 10 & 0,1 & 18 & 4,3 & 35 & 4,82 \\
\hline $\begin{array}{l}\text { San Carlos de } \\
\text { Guaroa }\end{array}$ & 7 & 427 & 2 & 14,05 & 11 & 4,9 & 11 & 11,3 & 9 & 0 & 15 & 9,2 & 16 & 6,34 \\
\hline $\begin{array}{l}\text { San Juan de } \\
\text { Arama }\end{array}$ & 2 & 767 & 3 & 66,50 & 10 & 60,3 & 9 & 17,4 & 9 & 8,5 & 15 & 11,4 & 17 & 22,71 \\
\hline San Juanito & 3 & 53,4 & 6 & 55,10 & 9 & 50,4 & 9 & 39,9 & 9 & 38,1 & 15 & 42,4 & 5 & 43,41 \\
\hline San Martín & 14 & 17,6 & 8 & 36,83 & 22 & 23,2 & 51 & 21,7 & 33 & 12,4 & 31 & 4,9 & 32 & 9,78 \\
\hline Uribe* & 3 & 66 & 0 & & 8 & 5,5 & 7 & 19,4 & 11 & 18,6 & 13 & 11,1 & 18 & 16,30 \\
\hline Vistahermosa & 7 & 225 & 7 & 17,90 & 12 & 16,4 & 12 & 40,7 & 9 & 11,5 & 17 & 27,1 & 16 & 33,16 \\
\hline $\begin{array}{l}\text { Villavicencio } \\
\text { EAAV }\end{array}$ & 18 & 3,9 & 24 & 0,72 & 32 & 0,9 & 97 & 0,2 & 97 & 0 & 110 & 1,8 & 84 & 0,25 \\
\hline $\begin{array}{l}\text { Villavicencio } \\
\text { Otros urbanos }\end{array}$ & & & 43 & 61,01 & 48 & 56,8 & & 26,046 & 71 & 42,3 & 82 & 40,9 & 103 & 33,52 \\
\hline Total muestras & 185 & & 190 & & 439 & & 493 & & 507 & & 732 & & 780 & \\
\hline $\begin{array}{l}\text { Total muestras } \\
\text { periodo }\end{array}$ & 3326 & & & & & & & & & & & & & \\
\hline $\begin{array}{l}\text { IRCApp } \\
\text { promedio }\end{array}$ & & 44,70 & & 34,96 & & 3226 & & 26,05 & & 23,10 & & 23,74 & & 25,89 \\
\hline
\end{tabular}

Tabla 3. Clasificación de los niveles de riesgo y concepto de la calidad de agua para consumo humano.

\begin{tabular}{lcc}
\hline \multicolumn{1}{c|}{ Nivel de riesgo } & IRCApp & Concepto de calidad \\
\hline Sin Riesgo & $0-5$ & Apta para consumo humano \\
Bajo & $5,1-14$ & No apta para consumo humano \\
Medio & $14,1-35$ & No apta para consumo humano \\
Alto & $35,1-80$ & No apta para consumo humano \\
Inviable Sanitariamente & $80,1-100$ & No apta para consumo humano \\
\hline
\end{tabular}


Tabla 4. Niveles de riesgo de los acueductos de las áreas urbanas en el periodo de 2008 a 2014.

\begin{tabular}{|c|c|c|c|c|c|c|c|}
\hline \multirow{2}{*}{$\begin{array}{l}\text { Acue ductos } \\
\text { Cabeceras } \\
\text { Municipales }\end{array}$} & \multicolumn{7}{|c|}{ Nivel de riesgo por IRCApp promedio anual } \\
\hline & 2008 & 2009 & 2010 & 2011 & 2012 & 2013 & 2014 \\
\hline Acacias & Bajo & Bajo & Sin riesgo & Bajo & Bajo & Sin riesgo & Bajo \\
\hline Barranca & Alto & Sin riesgo & Medio & Medio & Medio & Medio & Bajo \\
\hline Cabuyaro & Alto & Sin riesgo & Alto & Medio & Alto & Medio & Medio \\
\hline Castilla & Bajo & Medio & Alto & Bajo & Bajo & Bajo & Sin riesgo \\
\hline Cubarral & Alto & Alto & Alto & Alto & Alto & Alto & Inviable \\
\hline Cumaral & Alto & Sin riesgo & Bajo & Bajo & Sin riesgo & Sin riesgo & Bajo \\
\hline El Calvario & Alto & Alto & Alto & Alto & Alto & Alto & Alto \\
\hline El Castillo & Alto & Sin riesgo & Alto & Alto & Alto & Alto & Inviable \\
\hline El Dorado & Alto & Alto & Medio & Alto & Alto & Medio & Bajo \\
\hline Fuentedeoro & Alto & Medio & Medio & Bajo & Bajo & Sin riesgo & Bajo \\
\hline Granada & Alto & Alto & Inviable & Alto & Medio & Medio & Medio \\
\hline Guamal & Alto & Medio & Bajo & Bajo & Bajo & Sin riesgo & Bajo \\
\hline La Macarena & Alto & Sin riesgo & Medio & Medio & Alto & Medio & Medio \\
\hline Lejanías & Alto & Sin riesgo & Bajo & Medio & Medio & Medio & Inviable \\
\hline Mapiripán & Alto & Alto & Alto & Medio & Bajo & Medio & Bajo \\
\hline Mesetas & Alto & Sin riesgo & Alto & Alto & Alto & Inviable & Alto \\
\hline Puerto Concordia & Medio & Sin riesgo & Bajo & Bajo & Bajo & Bajo & Bajo \\
\hline Puerto Gaitán & Alto & Sin riesgo & Medio & Medio & Bajo & Medio & Medio \\
\hline Puerto Lleras & Alto & Sin riesgo & Medio & Sin riesgo & Sin riesgo & Bajo & Bajo \\
\hline Puerto López & Alto & Alto & Alto & Alto & Alto & Alto & Alto \\
\hline Puerto Rico & Alto & Sin riesgo & Sin riesgo & Sin riesgo & Sin riesgo & Bajo & Sin riesgo \\
\hline Restrepo & Medio & Sin riesgo & Sin riesgo & Bajo & Sin riesgo & Sin riesgo & Sin riesgo \\
\hline San Carlos de Guaroa & Alto & Bajo & Sin riesgo & Bajo & Sin riesgo & Bajo & Bajo \\
\hline San Juan de Arama & Alto & Alto & Alto & Medio & Bajo & Bajo & Medio \\
\hline San Juanito & Alto & Alto & Alto & Alto & Alto & Alto & Alto \\
\hline San Martin & Medio & Alto & Medio & Medio & Bajo & Sin riesgo & Bajo \\
\hline Uribe & Alto & Sin riesgo & Bajo & Medio & Medio & Bajo & Medio \\
\hline Villavicendo EAAV & Sin riesgo & Sin riesgo & Sin riesgo & Sin riesgo & Sin riesgo & Sin riesgo & Sin riesgo \\
\hline Villavicendo Otros urbanos & Sin riesgo & Alto & Sin riesgo & Medio & Alto & Alto & Medio \\
\hline Vistahermosa & Medio & Medio & Medio & Alto & Bajo & Medio & Medio \\
\hline $\begin{array}{l}\text { Código de colores por } \\
\text { nivel de riesgo: }\end{array}$ & Sin riesgo & Bajo & Medio & Alto & Inviable* & $\begin{array}{l}{ }^{*} \text { Invi } \\
\text { sanitari }\end{array}$ & $\begin{array}{l}\text { iable } \\
\text { amente }\end{array}$ \\
\hline
\end{tabular}


Tabla 5. Resumen niveles de riesgo por medición de los acueductos de las áreas urbanas en el periodo de 2008 a 2014.

\begin{tabular}{l|r|r}
\hline \multicolumn{1}{c|}{$\begin{array}{c}\text { Nivel de riesgo por } \\
\text { medición }\end{array}$} & No de registros & $\%$ \\
\hline Sin Riesgo & 29 & $14,7 \%$ \\
Bajo & 44 & $22,3 \%$ \\
Medio & 49 & $24,9 \%$ \\
Alto & 70 & $35,5 \%$ \\
Inviable Sanitariamente & 5 & $2,5 \%$ \\
Totales & 197 & $100 \%$ \\
\hline
\end{tabular}

Tabla 6. Distribución de los IRCApp promedio anual por acueducto de acuerdo con el intervalo de confianza de la media y valores máximos y mínimos.

\begin{tabular}{|c|c|c|c|c|}
\hline \multirow{2}{*}{ Acueducto } & \multirow{2}{*}{$\begin{array}{l}\text { Desviación } \\
\text { estándar }\end{array}$} & \multicolumn{3}{|c|}{ IRCA(\%)pp promedio anual } \\
\hline & & Máximo & Mínimo & Promedio \\
\hline EAAV - Villavicencio & 3,0 & 3,9 & 0,0 & 1,1 \\
\hline Restrepo & 6,6 & 18,0 & 0,1 & 5,4 \\
\hline Acacias & 3,0 & 11,0 & 2,4 & 6,9 \\
\hline Puerto Rico & 12,9 & 37,6 & 0,0 & 9,2 \\
\hline Puerto Concordia & 10,1 & 32,4 & 5,1 & 12,4 \\
\hline San Carlos de Guaroa & 14,0 & 42,7 & 0,0 & 12,6 \\
\hline Cumaral & 13,7 & 39,8 & 3,4 & 12,7 \\
\hline Castilla & 17,9 & 54,3 & 4,4 & 17,4 \\
\hline Puerto Lleras & 23,4 & 63,0 & 0,9 & 17,8 \\
\hline San Martín & 10,5 & 36,8 & 4,9 & 18,1 \\
\hline Guamal & 24,2 & 72,0 & 2,7 & 20,3 \\
\hline Fuentedeoro & 22,6 & 70,5 & 4,4 & 21,9 \\
\hline Uribe & 21,8 & 66,0 & 5,5 & 22,8 \\
\hline Puerto Gaitán & 10,3 & 42,4 & 13,9 & 24,1 \\
\hline Barranca & 8,7 & 36,7 & 14,1 & 24,2 \\
\hline Vistahermosa & 10,2 & 40,7 & 11,5 & 24,2 \\
\hline Lejanías & 26,5 & 81,0 & 0,0 & 29,0 \\
\hline El Dorado & 17,8 & 56,3 & 6,6 & 32,0 \\
\hline La Macarena & 8,5 & 44,9 & 23,1 & 32,2 \\
\hline Mapiripán & 27,7 & 79,8 & 10,0 & 36,5 \\
\hline San Juan de Arama & 29,0 & 76,7 & 8,5 & 37,6 \\
\hline Cabuyaro & 27,4 & 76,1 & 14,2 & 38,1 \\
\hline
\end{tabular}


Continuación Tabla 6. Distribución de los IRCApp promedio anual por acueducto de acuerdo con el intervalo de confianza de la media y valores máximos y mínimos.

\begin{tabular}{|c|c|c|c|c|}
\hline \multirow{2}{*}{ Acueducto } & \multirow{2}{*}{$\begin{array}{l}\text { Desviación } \\
\text { estándar }\end{array}$} & \multicolumn{3}{|c|}{ IRCA(\%)pp promedio anual } \\
\hline & & Máximo & Mínimo & Promedio \\
\hline Otros Vcio & 13,1 & 61,0 & 26,0 & 40,8 \\
\hline El Calvario & 5,3 & 54,3 & 39,8 & 45,2 \\
\hline San Juanito & 6,8 & 55,1 & 38,1 & 46,1 \\
\hline Puerto López & 8,5 & 64,2 & 36,8 & 47,0 \\
\hline Granada & 23,5 & 80,7 & 19,6 & 50,5 \\
\hline El Castillo & 10,5 & 82,0 & 51,6 & 69,4 \\
\hline Mesetas & 7,5 & 84,1 & 61,9 & 70,5 \\
\hline Cubarral & 9,8 & 80,6 & 51,8 & 70,6 \\
\hline
\end{tabular}

Tendencia del IRCApp acueductos cabeceras municipales del departamento del Meta

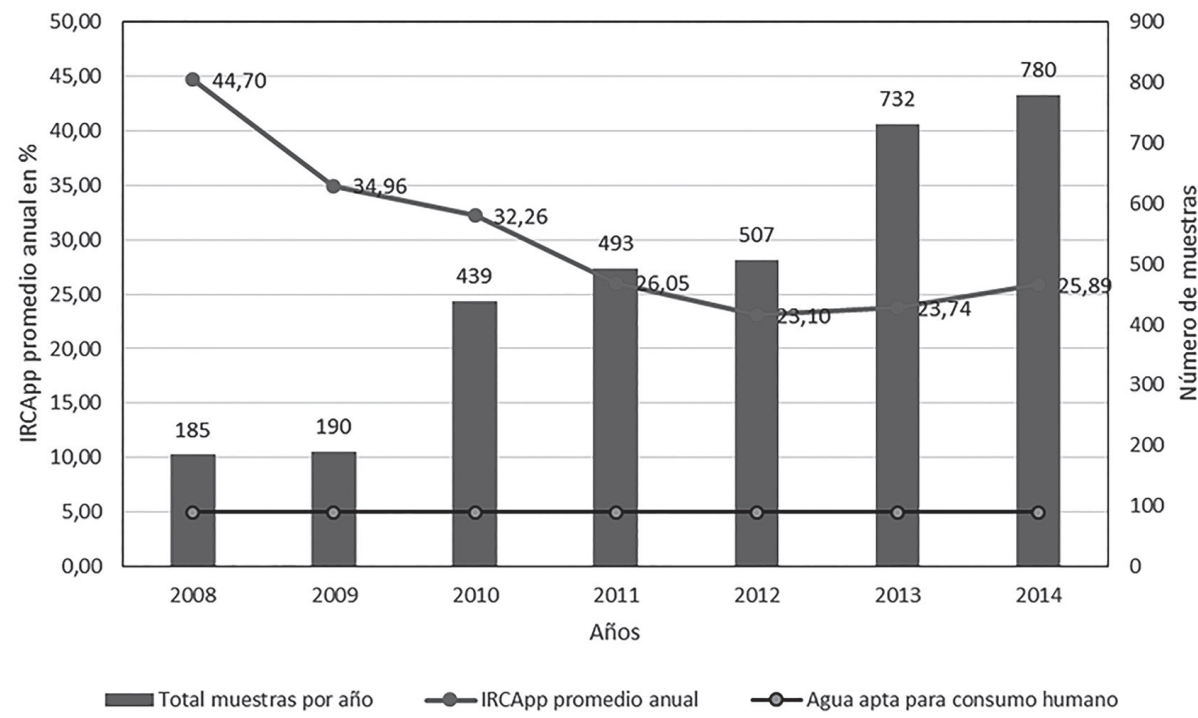

\begin{tabular}{lr|r|r|r|r|r|r}
\hline \multicolumn{1}{c|}{ Año } & $\mathbf{2 0 0 8}$ & $\mathbf{2 0 0 9}$ & $\mathbf{2 0 1 0}$ & $\mathbf{2 0 1 1}$ & $\mathbf{2 0 1 2}$ & $\mathbf{2 0 1 3}$ & $\mathbf{2 0 1 4}$ \\
\hline $\begin{array}{l}\text { Total muestras } \\
\text { por año }\end{array}$ & 185 & 190 & 439 & 493 & 507 & 732 & 780 \\
$\begin{array}{l}\text { IRCApp promedio } \\
\text { anual }\end{array}$ & 44,70 & 34,96 & 32,26 & 26,05 & 23,10 & 23,74 & 25,89 \\
\hline
\end{tabular}

Figura 1. Tendencia del IRCApp acueductos áreas urbanas del departamento del Meta. IRCApp: Índice de riesgo de la calidad del agua por persona prestadora.

28 Fuente: Los autores. Villavicencio, 2015. 


\title{
Niveles de riesgo por medición
}

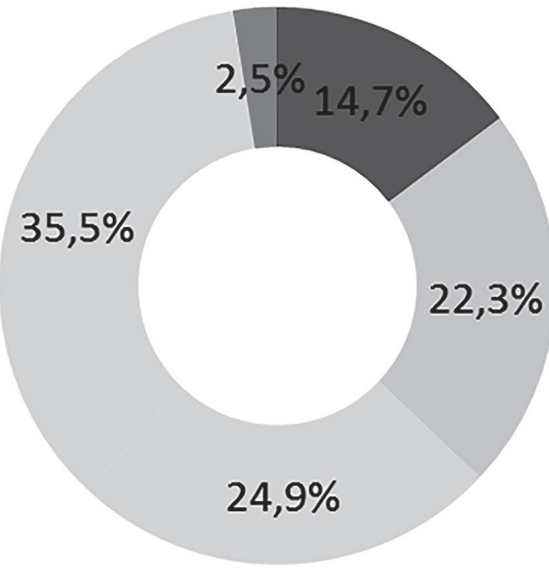

\author{
$\square$ Sin riesgo \\ Bajo \\ Medio \\ Alto
}

- Inviable Sanitariamente

Figura 2. Clasificación por nivel de riesgo de todas las mediciones.

Fuente: Los autores. Villavicencio, 2015.

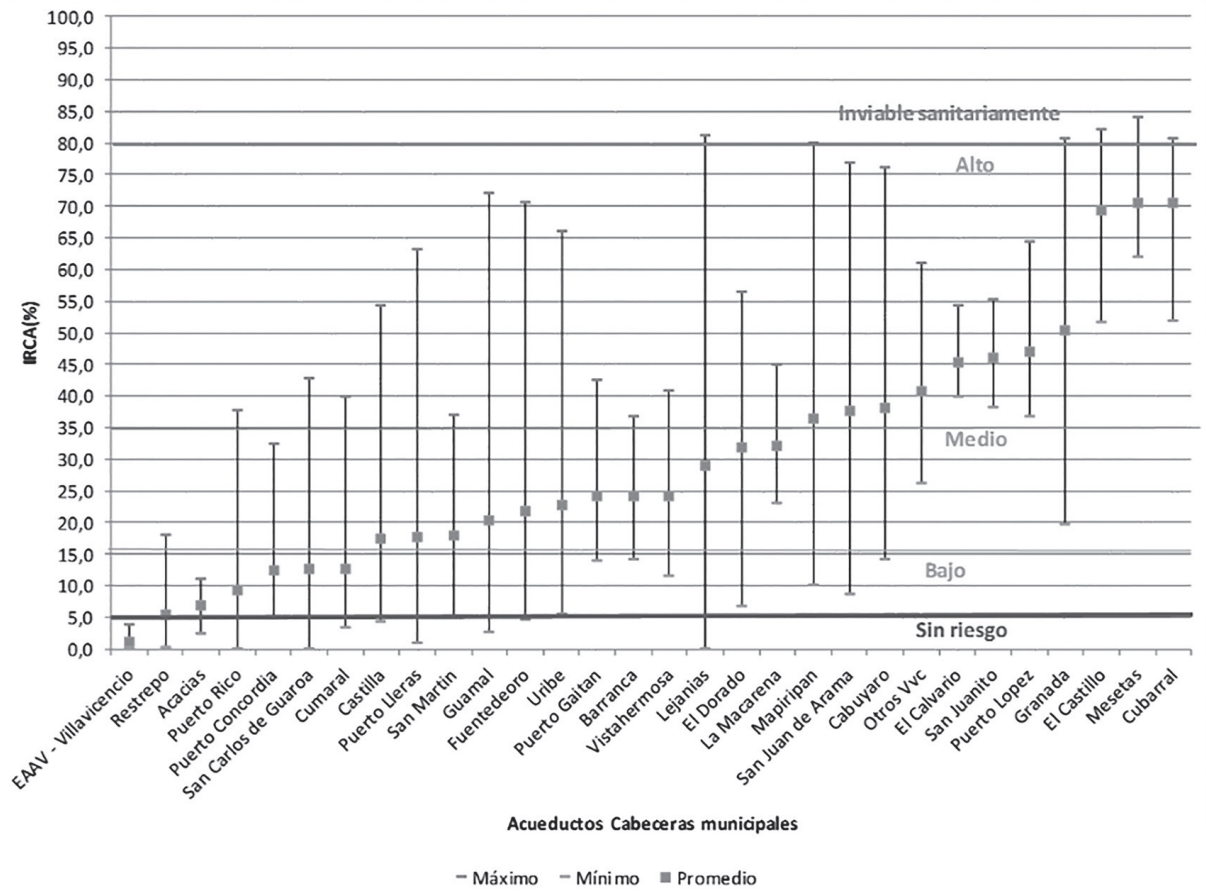

Figura 2. Distribución de los IRCApp por acueducto de acuerdo con el intervalo de confianza de la media y niveles de riesgo establecidos por la norma Colombiana Fuente: Los autores. Villavicencio, 2015. 
INVESTIGACIONES ANDINA No. 35, Vol. 19 